\title{
Considerações sobre Avaliação de
}

\section{Estabelecimentos de Saúde sob Gestão de OSS: - caso do Hospital Geral do Grajaú' Considerations on Evaluation of Health Facilities Management under the Model of Social Organizations of Health: the case of Grajaú General Hospital}

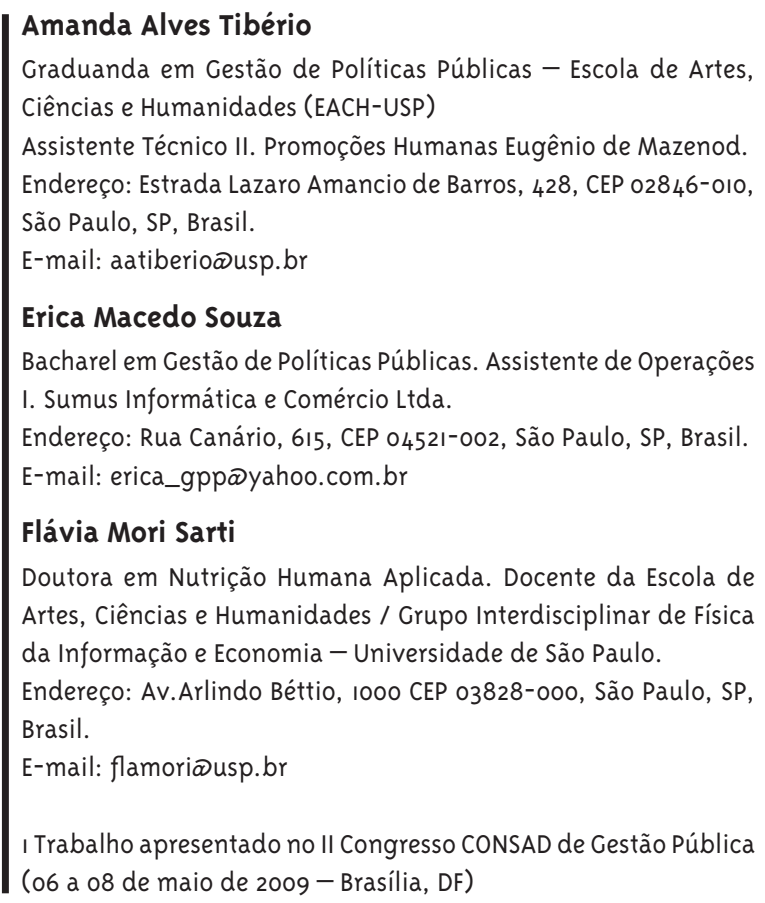

\section{Resumo}

Objetivos. A partir da década de 1990, em face de um cenário de limitações de recursos do Estado para financiamento de serviços essenciais à população, novas formas de administração pública começam a ser implementadas. Uma das principais formas adotadas refere-se à delegação da administração de hospitais públicos do estado de São Paulo às Organizações Sociais de Saúde (OSS). 0 artigo propõe um formato de avaliação da gestão das políticas públicas de saúde via OSS no município de São Paulo, a partir do caso do Hospital Geral do Grajaú. Procedimentos metodológicos. A avaliação concentrou-se em variáveis sócio-econômicas e demográficas da população local e sua articulação com variáveis estruturais da rede básica de saúde disponíveis; além de dados sobre infra-estrutura da instituição selecionada e relatórios da organização social de saúde no período entre 2004 e 2007. Resultados. A região apresenta déficit de unidades básicas de saúde, que gera sobrecarga de atendimentos de baixa complexidade no hospital. Os resultados da avaliação comparativa entre oferta e demanda por serviços públicos de saúde, a partir do perfil populacional da região e características de atendimentos realizados pela organização social, indicam que a unidade buscou adaptar-se às necessidades prioritárias da região. No entanto, verificou-se, também, que a implantação de um único hospital em uma região de alta vulnerabilidade social é insuficiente para suprir as necessidades da população, sendo necessária uma rede de unidades básicas de saúde para auxiliar na realização de atendimentos de baixa complexidade, 
permitindo focalização do atendimento hospitalar sobre atendimentos de média e alta complexidade. Palavras-chave: Políticas públicas de saúde; Gestão em saúde; Administração de recursos; Avaliação; Administração pública.

\section{Abstract}

Objectives. Since the 1990s, in light of a scenario of limited government resources to finance essential public services to address population needs, new public administration models have been implemented. One of the main models was the delegation of public hospitals management to Organizações Sociais de Saúde (OSS - Social Organizations of Health) in the state of São Paulo. The paper proposes a structure for the evaluation of public health policy management through OSS in the city of São Paulo, starting from the case of Grajaú General Hospital. Methods. Evaluation was centered on socio-economic and demographic variables from local population and their articulation with structural variables available from the primary healthcare network, as well as infrastructure data from the selected hospital and reports from the OSS during the period from 2004 to 2007. Results. The region presents a significant deficit in primary healthcare units, which results in overload of low complexity health services demand in the hospital. Results from the comparative evaluation between public health services' supply and demand in the region, crossing information on population profile and health services provided by the OSS, indicate that the hospital adapted its structure to meet the population's essential needs. Nevertheless, it was also verified that implementing one single hospital in a high social vulnerability region is insufficient to satisfy all the population's demands; thus, it is necessary to create a network of primary healthcare units to support hospital actions in public health, especially to assume low complexity health services, so that the hospital can focus on middle and high complexity health services.

Keywords: Health Public Policy; Health Management; Resources Management; Evaluation; Public Administration. 


\section{Introdução}

A expansão da rede hospitalar do estado de São Paulo, a partir da década de 1990, foi baseada em modelo concebido à luz de um novo paradigma de administração pública, representado pela Reforma do Estado. $O$ formato de gestão adotado no setor saúde estabeleceu um novo modelo de administração de equipamentos e serviços públicos, via delegação das operações relativas a novas instituições de saúde do Estado às Organizações Sociais de Saúde (OSS), através de um instrumento denominado contrato de gestão (Gomes, 2005).

A escolha do novo modelo de gestão baseou-se no argumento de que o governo não apresentava condições financeiras para arcar com responsabilidades referentes à contratação de recursos humanos para viabilização das atividades nos novos hospitais, sem que o gasto excedesse o percentual máximo indicado por lei. Ademais, apontava-se ineficiência do Estado no tocante às constantes demandas por adaptação e reformulação nos procedimentos burocráticos e processuais para atendimento das variadas demandas sociais existentes (Carneiro Jr. e Elias, 2003).

Assim, a geração de um novo instrumento de controle - o contrato de gestão contrato de gestão - viabilizou a delegação de atividades-fim à OSS, sem que o Estado seja eximido de responsabilidades relativas à fiscalização, controle e resultados obtidos em ações de saúde executadas por terceiros (Bresser-Pereira, 2008).

O presente artigo buscou avaliar o cenário em saúde da zona sul do município de São Paulo, particularmente o caso do Hospital Geral do Grajaú, a partir de variáveis sócio-econômicas e demográficas da população e sua articulação com variáveis estruturais da rede básica de saúde local; além de dados sobre infra-estrutura da instituição selecionada (recursos humanos e equipamentos) e relatórios da organização social de saúde no período de 2004 a 2007 .

\section{Políticas Públicas de Saúde no Estado de São Paulo}

Até meados do século XIX, as políticas de saúde no estado de São Paulo não apresentavam um perfil sis- temático, tendo como foco principal o atendimento a doentes psiquiátricos e o combate a epidemias. Tal perfil só foi modificado a partir de 1970, a partir do estabelecimento de políticas sistemáticas relativas à ampliação de leitos e atendimento a diferentes tipos de enfermidades (Gomes, 2005).

Dado o novo perfil de atendimento, o governo buscou ampliar o número de hospitais disponíveis à população, contudo, o sistema público hospitalocêntrico à época não oferecia qualidade nos serviços prestados, sendo que qualquer proposta de mudança no atendimento esbarrava não apenas nas dificuldades financeiras do Estado, mas também na burocracia para compra de materiais e contratação de recursos humanos, tornando lenta e ineficaz a adequação do serviço às demandas sociais (Ibañez e col., 2001).

Durante duas décadas, o governo do estado de São Paulo buscou implantar alguns modelos diferenciados de gestão de hospitais, como fundações privadas de apoio à administração de hospitais públicos e transformação de hospitais da administração direta em fundações públicas. Entretanto, em meados de 1981, o governo estabeleceu o Sistema Estadual de Saúde, buscando ampliar e melhorar a qualidade dos serviços oferecidos à população através do Programa Metropolitano de Saúde (PMS), que seguia as diretrizes da reforma sanitária: integração institucional, regionalização e hierarquização, modularização e gestão colegiada do sistema de saúde (Ibañez e col., 2001).

A implementação do PMS exigia recursos financeiros para reforma da infra-estrutura de saúde já existente e construção de novos equipamentos de saúde, além de contratação e capacitação de novos quadros de recursos humanos. Contudo, devido à crise fiscal da época, o governo optou em recorrer ao financiamento da Caixa Econômica Federal e Banco Internacional de Reconstrução e Desenvolvimento (BIRD). Tais recursos não foram suficientes para iniciar operacionalização de todos os hospitais recémconstruídos, um cenário que permaneceu vigente durante alguns anos sem que o governo conseguisse executar e implementar efetivamente o Programa Metropolitano de Saúde (Ibañez e col., 2001).

Somente após a instituição do marco regulatório da saúde na Constituição Federal de 1988 houve mudanças no cenário, visto que a saúde torna-se um 
direito de todos os cidadãos brasileiros, sendo dever do Estado garantir o acesso de maneira universal e igualitária. Quanto à regulação e controle da compra de serviços de saúde, a Constituição Federal, no Art. 199, parágrafo $1^{\circ}$, prevê que "as instituições privadas poderão participar de forma complementar do Sistema Único de Saúde, segundo diretrizes deste, mediante contrato de direito público ou convênio, tendo preferência às entidades filantrópicas e as sem fins lucrativos" (Brasil, 1988).

A década de 1990 é marcada por mudanças no papel do Estado, escassez de recursos e insatisfação dos cidadãos na prestação dos serviços públicos. Existia necessidade de criar um novo modelo de gestão pública capaz de otimizar o uso de tempo e recursos. Ressalta-se que os problemas na gerência da saúde não constituíam questões somente de âmbito local, mas eram parte de uma crise nacional cujas origens remontam aos primórdios do sistema público de saúde brasileiro, anteriores, portanto, à promulgação da Constituição Federal de 1988.

A formação das políticas de saúde no estado de São Paulo teve significativa influência dos movimentos sociais de saúde, cujas bases remontam à organização de associações de bairros e ao movimento sanitarista, na demanda por equipamentos públicos e melhor qualidade no atendimento em saúde. 0 Hospital do Geral do Grajaú foi resultado de prolongada luta do movimento social de saúde para instituição de políticas públicas de saúde consistentes, assim como fruto da Reforma do Estado, que resultou na expansão de equipamentos públicos de saúde para a periferia de São Paulo.

\section{Reforma do Estado}

Em diversas municipalidades brasileiras, a implantação do SUS foi adiada por diversos motivos, sendo a falta de recursos financeiros o principal problema enfrentado no âmbito da organização do sistema de saúde descentralizado - apesar da Lei 8.080/9o ter definido que os recursos ao estabelecimento do SUS seriam provenientes do Orçamento de Seguridade Social, sendo um repasse flexível, de acordo com o volume de arrecadação do governo federal. Em 1991, foram alteradas leis de repasse de recursos pelo governo federal aos estados e municípios, que vinculava repasse ao perfil populacional, fatores epidemiológicos e demográficos e, principalmente, à produção de serviços de saúde.

O governador do estado de São Paulo à época, Mário Covas (1995-1998), finalizou as obras de construção de diversos estabelecimentos de saúde, porém, havia ainda um obstáculo a ser vencido: a limitação de recursos financeiros para contratação de funcionários, tendo em vista a crise financeira do período, assim como as barreiras jurídicas, como a Lei Complementar 82/95 (Lei Camata), a Lei 8666/93 (Lei de Licitações) e a legislação de saúde vigente em São Paulo, que proibia a terceirização da gerência de hospitais públicos estaduais e a cobrança pelos serviços oferecidos a particulares (Rinne, 2007).

Perante as limitações jurídicas quanto a gastos dos entes da federação em recursos humanos e o questionamento quanto à legalidade das fundações de apoio, o governo do estado de São Paulo buscou estudar novas formas possíveis de administração de equipamentos de saúde, tendo como perspectiva ampliar a disponibilidade de rede de assistência à saúde existente, sem, contudo, endividar ainda mais o Estado (Rinne, 2007).

Paralelamente, a discussão sobre a Reforma do Estado obteve maior repercussão a partir de 1995, quando foi apresentado o esboço do plano diretor de Reforma do Estado, que, segundo Bresser-Pereira (2001), busca incorporar a lógica de mercado à administração pública. A reforma da estrutura de administração pública constituiu o caminho adotado pelo governo brasileiro para enfrentar vários problemas administrativos, em especial a crise fiscal e a necessidade de redução do aparato estatal, delegando e repassando atividades e funções a setores de mercado e organizações sem fins lucrativos (Costa e Melo, 1998).

As mudanças implementadas via Reforma do Estado apresentavam como principais objetivos: redução do déficit público, de forma a aumentar a capacidade financeira do Estado; diminuição dos custos e melhoria da qualidade dos serviços prestados pelo governo; ampliação da participação da sociedade nos processos decisórios relativos a tais serviços; e desenvolvimento da eficácia e efetividade dos gestores de alto escalão do governo via controle de resultados (Modesto, 1997). 
O novo paradigma de administração pública exposto na Reforma do Estado constituiu a base das reflexões na busca de uma solução à crise enfrentada pelo governo, sendo a principal fonte de inspiração para criação do mecanismo de delegação de atividades-fim às organizações sociais, que possibilitou a operacionalização dos hospitais recém-inaugurados no estado de São Paulo, visto que as despesas com recursos humanos destes hospitais passariam a ser incluídas contabilmente como outras despesas (Rinne, 2007).

A delegação de atividades-fim buscava reorientar ações estatais para maior eficiência e melhor qualidade dos serviços prestados, permitindo maior flexibilidade gerencial em áreas de recursos humanos e materiais e focalizando prioritariamente resultados, qualidade, satisfação do cidadão-usuário e adequação à ocorrência de mudanças na demanda por serviços de saúde (Bazilli e Montenegro, 2003).

O modelo gerencial adotado eximia o Estado quanto à exoneração dos gastos públicos com pagamento de recursos humanos, tendo em vista que, ao delegar a função de administração a outro ator, a despesa deixa de ser classificada como despesa com recursos humanos. Uma vez que os funcionários não são contratados diretamente pelo governo, os gastos apresentam caráter de "outras despesas", o que permite, assim, respeitar os limites fiscais impostos por lei (Rinne, 2007).

Assim, face ao cenário de crise fiscal e novos conceitos introduzidos na administração pública brasileira, há uma redefinição de papel do governo federal e seus entes quanto à provisão de bens e serviços à sociedade. As Organizações Sociais (OS) tornam-se as principais instituições de redefinição do tamanho do Estado - "ou seja, a execução de atividades antes consideradas de caráter estatal passa a ser realizada por instituições sem fins lucrativos" (Carneiro Jr. e Elias, 2003 p. 208) -, marcando o início da efetiva participação de novos atores sociais na prestação de serviços à sociedade.

Embora utilizem recursos públicos para realização de suas atividades, as Organizações Sociais configuram-se como pessoas jurídicas de direito privado sem fins lucrativos, que independem de concessão ou permissão do poder público, sendo, contudo, reconhecidas, fiscalizadas e fomentadas pelo Estado (Modesto, 1997). Portanto, configuramse como participantes do terceiro setor que não podem gerar lucros e têm como obrigação reinvestir o excedente financeiro gerado em suas atividades na própria organização.

A delegação dos serviços de saúde do governo do estado de São Paulo às Organizações Sociais exigiu editar a Lei Complementar 846/98, que instituiu a possibilidade de qualificação de organizações sem fins lucrativos como Organizações Sociais de Saúde (OSS), desde que atendessem a alguns critérios, tais como: comprovação de experiência de, no mínimo, cinco anos na administração de serviços de saúde; submissão de dados de contabilidade à análise do Tribunal de Contas do Estado; e atendimento direcionado somente a pacientes do Sistema Único de Saúde. A lei também regulamenta a criação do contrato de gestão, cuja finalidade reside em constituir o instrumento de regulação da parceria entre poder público e OSS, tendo como base critérios técnicos bem estabelecidos: definição das metas e resultados, caracterização do modelo de assistência à saúde a ser desenvolvido; e vinculação da remuneração à transparência no fornecimento de dados (São Paulo, 1998).

A delegação das atividades-fim em saúde para Organizações Sociais possibilitou ao governo de São Paulo a rápida operacionalização dos novos hospitais e a ampliação do número de equipamentos e serviços oferecidos à população. Atualmente, dados da FGV/ UNESCO (2008), revelam que o estado de São Paulo possui 21 hospitais, três ambulatórios, duas farmácias e três laboratórios de análises clínicas sob contrato de gestão com Organizações Sociais de Saúde.

O contrato de gestão desenvolvido pelo governo do estado de São Paulo impõe ao poder público as seguintes obrigações: planejamento dos serviços; ampliação ou redução da produção assistencial; melhoria da qualidade dos serviços prestados; previsão orçamentária dos recursos desembolsados; controle das atividades desenvolvidas; e transparência na gestão dos recursos públicos. Quanto às responsabilidades das Organizações Sociais de Saúde, são exigidos: pontualidade, exatidão e confiabilidade dos dados gerados; avaliação quantitativa e qualitativa contínua; relação transparente e de confiança; e a qualificação e constante aprimoramento das 
atividades. Contudo, existe determinado grau de flexibilidade no tocante à efetivação das metas e resultados estipulados pelo poder público (FGV/ UNESCO, 2008).

A permanente autonomia das OSS em relação aos processos internos, em contraposição aos resultados a serem apresentados, gera comprometimento entre duas partes - Estado e Organização Social -, que, por sua vez, implica na responsabilização e prestação de contas sobre serviços realizados. No entanto, o contrato de gestão não representa uma relação hierárquica, somente uma relação de parceria que exige negociação do contrato entre as partes envolvidas (Pacheco, 2004).

Na última década, novas reformulações foram imputadas ao contrato de gestão, buscando-se o aprimoramento do referido instrumento através das seguintes mudanças: introdução de indicadores de avaliação diferenciados de acordo com modalidade de atendimento - "porta aberta" (hospitais que atendem à demanda espontânea da sociedade) ou "porta fechada" (hospitais que funcionam com referência restrita e organizações que possuem ambulatórios de especialidades servindo à rede pública) -; inclusão de cláusulas que estabelecem um teto máximo de $70 \%$ do orçamento destinado ao hospital sob forma de despesas com recursos humanos; modificação expressiva no repasse do orçamento anual - condicionado a doze parcelas mensais iguais de $90 \%$ do orçamento anual, sendo que os 10\% restantes são condicionados à avaliação de desempenho dos indicadores estipulados pela Secretaria de Estado da Saúde de São Paulo (SES/SP) -; e criação de indicadores de avaliação como: qualidade de informação, média de permanência em internação e atenção ao usuário (Carneiro Jr. e Elias, 2003).

\section{Avaliação da Gestão em Saúde sob OSS: o caso do Hospital Geral do Grajaú}

A partir de uma análise prévia da nova proposta administrativa do governo no campo das políticas de saúde, buscou-se avaliar o processo de publicização da saúde no estado de São Paulo pela realização de um estudo de caso: o Hospital Geral do Grajaú, localizado na periferia da zona sul da cidade de São
Paulo. A escolha do estudo de caso partiu de estudo do perfil sócio-demográfico da região atendida pelo hospital.

A avaliação do impacto da implementação de um hospital gerenciado por uma Organização Social de Saúde em um contexto específico de estudo de caso deve basear-se na observação do contexto no qual o hospital foi implantado e as características da região.

O conceito de avaliação utilizado no contexto do presente artigo refere-se ao processo de comparação de resultados potenciais (avaliação ex-ante ou a priori) ou efetivos (avaliação ex-post ou a posteriori) de um conjunto de ações em relação à situação vigente ou a um padrão-ouro, de forma a verificar se os objetivos propostos são alcançados nos moldes de eficiência e efetividade ou eficácia planejados (Sarti e Campino, 2010).

Optou-se pela abordagem de avaliação ex-post do cenário de oferta (infra-estrutura e recursos humanos e materiais do Hospital Geral do Grajaú) e demanda (perfil sócio-demográfico e epidemiológico da população local) de serviços de saúde no entorno do estabelecimento, de forma a verificar se os objetivos propostos da política pública de saúde em São Paulo têm sido alcançados. No caso, a política pública de parceria na gestão de hospitais entre Estado e OSS tem como objetivo atender às demandas populacionais de forma flexível e adaptativa.

O Hospital Geral do Grajaú situa-se na região de Capela do Socorro, composta por três distritos (Socorro, Cidade Dutra e Grajaú) com 656.695 habitantes no ano de 2007, de acordo com estimativa populacional realizada pela Fundação SEADE (2008), sendo considerada a região mais populosa da cidade de São Paulo. 0 funcionamento da unidade hospitalar teve início no ano de 1999, no entanto, ressalta-se que o contrato de gestão com a primeira organização social gerenciadora, a Organização Santamarense de Educação e Cultura (OSEC) foi assinado ao final de outubro de 1998.

O hospital localiza-se no distrito do Grajaú, que constitui o distrito mais populoso da região, apresentando em torno de 420 mil habitantes no ano de 2007 (Fundação SEADE, 2008), assim, a avaliação empreendida no presente artigo concentrou-se na região de abrangência do hospital. 
O distrito apresenta do Grajaú maior concentração de população do sexo feminino do que masculino. A população masculina é maior apenas na faixa etária de zero a 14 anos de idade, entretanto, pode-se observar que ocorreu queda na diferença entre população masculina e feminina na região durante o período em questão (Tabela 1).

A população do distrito de Grajaú foi distribuída em três grupos etários, de forma a caracterizar a distribuição demográfica entre crianças e jovens, adultos e idosos. A partir dos dados demográficos do distrito de abrangência do Hospital Geral do
Grajaú, destaca-se que, em 2007, cerca de 40\% dos habitantes da região estavam na faixa etária entre zero e 19 anos de idade, ou seja, uma população predominantemente jovem. 0 segundo grupo etário apresenta a maior parte da população economicamente ativa na faixa entre vinte a 59 anos de idade, correspondendo a $56 \%$ da população, especialmente concentrados na faixa dos vinte aos 34 anos de idade, e o terceiro grupo etário, que compreende os indivíduos com idade igual ou superior a 60 anos de idade (idosos), representava pouco mais de $4 \%$ dos habitantes da região.

Tabela I - População do distrito de Grajaú, segundo gênero e faixa etária. São Paulo, 2004-2007

\begin{tabular}{|c|c|c|c|c|c|c|c|c|}
\hline \multirow{2}{*}{ Faixa Etária } & \multicolumn{2}{|c|}{2004} & \multicolumn{2}{|c|}{2005} & \multicolumn{2}{|c|}{2006} & \multicolumn{2}{|c|}{2007} \\
\hline & M & $\mathrm{F}$ & M & $\mathrm{F}$ & M & $\mathrm{F}$ & M & $\mathrm{F}$ \\
\hline 024 & 22.610 & 21.827 & 23.630 & 22.735 & 23.708 & 22.619 & 23.825 & 22.529 \\
\hline 529 & 20.149 & 19.924 & 20.957 & 20.705 & 21.893 & 21.362 & 22.864 & 22.020 \\
\hline $10 \mathrm{a} 14$ & 17.782 & 17.490 & 18.029 & 17.664 & 18.756 & 18.177 & 19.500 & 18.683 \\
\hline 15 a 19 & 17.768 & 18.646 & 17.880 & 18.580 & 18.132 & 18.602 & 18.392 & 18.609 \\
\hline Crianças/Jovens & 78.309 & 77.887 & 80.496 & 79.684 & 82.489 & 80.760 & 84.581 & 81.841 \\
\hline 20 a 24 & 18.513 & 19.632 & 18.873 & 19.932 & 19.006 & 19.702 & 19.117 & 19.459 \\
\hline 25 a 29 & 18.455 & 19.740 & 19.099 & 20.501 & 19.443 & 20.576 & 19.793 & 20.670 \\
\hline 30 a 34 & 16.956 & 17.946 & 17.554 & 18.633 & 18.179 & 19.140 & 18.799 & 19.646 \\
\hline 35 a 39 & 14.714 & 15.389 & 15.319 & 15.967 & 15.888 & 16.404 & 16.451 & 16.876 \\
\hline 40 a 44 & 12.711 & 13.434 & 13.368 & 14.124 & 13.918 & 14.478 & 14.476 & 14.869 \\
\hline 45 a 49 & 10.246 & 10.683 & 10.903 & 11.371 & 11.475 & 11.803 & 12.044 & 12.263 \\
\hline 50 a 54 & 7.770 & 7.835 & 8.337 & 8.449 & 8.865 & 8.886 & 9.417 & 9.342 \\
\hline 55 a 59 & 5.177 & 5.158 & 5.670 & 5.659 & 6.083 & 6.028 & 6.506 & 6.389 \\
\hline Adultos & 104.542 & 109.817 & 109.123 & 114.636 & 112.857 & 117.017 & 116.603 & 119.514 \\
\hline 60 a 64 & 2.770 & 3.178 & 2.963 & 3.383 & 3.229 & 3.665 & 3.534 & 3.933 \\
\hline 65 a 69 & 1.715 & 2.190 & 1.854 & 2.345 & 1.964 & 2.468 & 2.081 & 2.569 \\
\hline 70 a 74 & 1.008 & 1.419 & 1.081 & 1.509 & 1.158 & 1.608 & 1.228 & 1.684 \\
\hline+75 & 1.010 & 1.733 & 1.103 & 1.878 & 1.168 & 1.976 & 1.237 & 2.075 \\
\hline Idosos & 6.503 & 8.520 & 7.001 & 9.115 & 7.519 & 9.717 & 8.080 & 10.261 \\
\hline Total & 189.354 & 196.224 & 196.620 & 203.435 & 202.865 & 207.494 & 209.264 & 211.616 \\
\hline
\end{tabular}

Fonte: Fundação SEADE (2008). 
O Índice Paulista de Vulnerabilidade Social (IPVS), desenvolvido pela Fundação SEADE, analisa a situação de vulnerabilidade dos distritos do município de São Paulo a partir de dados do Censo Demográfico de 200o, combinando vários indicadores sociais, tais como anos de estudo, renda, idade, entre outros. Através do IPVS, foi possível caracterizar o distrito do Grajaú como pertencente aos grupos de alta e muito alta vulnerabilidade social (IPVS 5 e 6 , respectivamente), ou seja, a região de abrangência do hospital apresenta alta concentração de população com baixo poder aquisitivo, baixa escolaridade, predominância de chefes de família jovens e significativa presença de crianças e jovens.

O perfil epidemiológico da região apresenta problemas de saúde específicos a populações muito jovens residentes em áreas de baixo desenvolvimento sócio-econômico. Uma importante especificidade geográfica do distrito do Grajaú refere-se à sua localização em uma área de manancial. Adicionalmente, caracteriza-se por alta densidade populacional com ampla presença de moradias e construções irregulares, sendo considerado um bolsão de pobreza no município. De acordo com Barban (2007), a região de Capela do Socorro apresenta um déficit de doze Unidades Básicas de Saúde (UBS) - deficiência justificada pelo poder público como resultante das características geográficas da região, que dificultam a construção de novas estruturas de apoio à infra-estrutura de saúde já existente e, conseqüentemente, prejudicam o trabalho de rede do hospital.

A partir da caracterização global da região da Capela do Socorro, é possível verificar que há 15 UBS para 656.695 habitantes (Barban, 2007). De acordo com padrões estabelecidos pela Organização Mundial de Saúde (Barban, 2007) - que prevê uma UBS para cada 20 mil habitantes -, o adequado atendimento da população local requer construção de, no mínimo, 17 unidades básicas de saúde adicionais, além de contratação de recursos humanos, aquisição de medicamentos e compra de equipamentos. Somente considerando a população residente no distrito do Grajaú no ano de 2007 (420 mil habitantes), seriam necessárias 21 unidades básicas de saúde para prover atendimento em saúde à população local.
O Hospital Geral do Grajaú efetua atendimentos por livre demanda, ou seja, atende diretamente aos cidadãos sem prévio encaminhamento da UBS. Ademais, o hospital constitui uma das poucas unidades hospitalares que apresenta capacidade instalada para realização de exames de elevado custo e alta especificidade, tais como: mamografia, ressonância magnética e tomografia computadorizada. Assim, o hospital do Grajaú é referência na região para tais tipos de exames, gerando significativa demanda pela população local, de forma que indivíduos residentes na região não necessitem deslocamento a hospitais centrais.

Face ao cenário sócio-econômico e demográfico apresentado, o déficit de infra-estrutura para atendimentos básicos em saúde na região sobrecarrega a unidade hospitalar com atendimentos e exames de rotina direcionados a pacientes externos ao entorno do hospital, isto é, pacientes encaminhados para realização de atividades de Serviço de Apoio Diagnóstico e Terapêutico (SADT) por outros serviços de saúde da região. A administração do hospital busca cumprir uma de suas principais funções - oferecer serviços de média e alta complexidade para a população da periferia - sem sobrecarregar outros equipamentos públicos de saúde. De acordo com dados do CNES-DataSUS apenas um serviço é terceirizado, a lavanderia, pois o serviço de manutenção de equipamentos não é totalmente terceirizado.

Segundo o Cadastro Nacional de Estabelecimentos de Saúde (DATASUS, 20o8), o quadro completo de recursos humanos no hospital é composto por 1.005 profissionais de saúde - entre médicos, enfermeiros, técnicos e auxiliares de enfermagem, que atuam diretamente no atendimento à população -, e 342 funcionários em outras funções.

Avaliando-se somente o quadro de profissionais da saúde, as especialidades médicas que apresentavam maior número de profissionais no ano de 2008 são (DATASUS, 2008): clínico geral (113), pediatria (56), ginecologista/obstetra (35) e ortopedia (26); o que corresponde ao perfil da população e aos principais tipos de atendimento realizados no hospital. Cerca de 40\% da população é jovem, sendo a maioria do sexo feminino. O perfil do quadro profissional em saúde do hospital é adequadamente adaptado às necessidades e ao perfil demográfico da região, pois 
nota-se que as principais especialidades atendem ao tipo de população majoritariamente presente na região.

O número de leitos também apresenta relação direta com perfil demográfico da região (DATASUS, 2008): no ano de 2008, havia significativo número de leitos nas áreas de clínica pediátrica (70) e UTI neonatal e pediátrica (24), no entanto, o atendimento geral da população ainda apresenta déficit importante, pois, de acordo com padrões da Organização Mundial da Saúde (Barban, 2007), é recomendável que estejam disponíveis quatro leitos por mil habitantes, no caso do hospital do Grajaú há somente 276 leitos, ou seja, o,28 leitos por mil habitantes.

Assim, observa-se que há direta relação entre perfil da população e o tipo de oferta de atendimento em saúde, a partir da comparação entre características básicas de capacidade de atendimento do Hospital Grajaú e dados demográficos locais. É possível apontar que a organização social gerenciadora do Hospital Geral do Grajaú busca adequar a prestação de serviços às demandas locais, apesar da sobrecarga no atendimento gerada pelo déficit de UBS no distrito.

Os dados de relatórios referentes ao período de 2004 a 2007 produzidos pela Organização Social de Saúde (OSS) gerenciadora do Hospital Geral do
Grajaú (São Paulo, 2008) foram utilizados na avaliação da evolução da produção de serviços em termos de atendimentos à população do distrito de abrangência do hospital, sendo divididos em: internação hospitalar (clínica médica, cirúrgica, obstétrica e pediátrica), atendimento ambulatorial, atendimento de urgência, serviço de apoio diagnóstico e terapêutico (SADT exame externo).

Observa-se, a partir dos dados de internações (Tabela 2), que a modalidade de clínica médica representa aproximadamente $20 \%$ dos atendimentos de internação, sendo caracterizado como um tipo de internação que constitui a primeira etapa do atendimento hospitalar aos pacientes. Contudo, em torno de $34 \%$ dos atendimentos realizados ocorre na área cirúrgica, tendo em vista o fato de ser o único hospital da região e apresentar ampla gama de especialidades, o que justifica a alta demanda da modalidade.

A clínica obstétrica realiza em torno de 3.000 atendimentos por ano, cerca de $20 \%$ das internações realizadas pelo hospital. A população feminina na faixa de 15 a 49 anos de idade - faixa etária que concentra maior parte da fase reprodutiva da mulher -, representa cerca de 30\% da população total do distrito, o que destaca a importância dos atendimentos na modalidade obstétrica à população local.

\begin{tabular}{|c|c|c|c|c|}
\hline Especialidade & 2004 & 2005 & 2006 & 2007 \\
\hline Clínica Médica & 2.671 & 2.783 & 3.252 & 3.169 \\
\hline Clínica Cirúrgica & 4.538 & 5.056 & 4.862 & 5.184 \\
\hline Clinica Obstétrica & 2.972 & 2.834 & 3.154 & 3.168 \\
\hline Clinica Pediátrica & 3.515 & 4.087 & 4.304 & 4.057 \\
\hline Total & 13.696 & 14.760 & 15.572 & 15.578 \\
\hline
\end{tabular}

Fonte: Baseado em dados de São Paulo (2008).

A região de abrangência do hospital é caracterizada pela presença de população predominantemente jovem - cerca de $40 \%$ da população apresenta menos de 19 anos de idade, sendo $11 \%$ da população total do distrito na faixa etária de zero a quatro anos, no ano de 2007 - a área pediátrica constitui uma seção de atendimento em saúde extremamente necessária à população local. As internações em clínica pediátrica representam o segundo maior volume de atendimentos realizados, em torno de $26 \%$ do total de internações hospitalares realizadas.

Quanto ao atendimento ambulatorial, registrouse acentuado incremento na demanda local durante o período compreendido entre os anos de 2004 e 2007. Uma das possíveis causas pode ser o déficit de unidades básicas de saúde na região (Tabela 3). 
Tabela 3 - Atendimentos ambulatoriais no Hospital Geral do Grajaú, segundo tipo de atendimento. São Paulo, 2004-2007

\begin{tabular}{lcccc} 
Tipo de atendimento & 2004 & 2005 & 2006 & 2007 \\
Atendimento ambulatorial & 61.191 & 47.870 & 124.585 & 119.689 \\
Atendimento de urgência & 325.345 & 198.815 & 302.645 & 281.074 \\
SADT (Exame externo) & 352.217 & 283.953 & 1.373 .051 & 416.412 \\
\hline
\end{tabular}

Fonte: Baseado em dados de São Paulo (2008).

Como enfatizado anteriormente, o distrito de Grajaú necessita de pelo menos 21 UBS para atender adequadamente à população local, sendo que a totalidade de UBS na região de Capela do Socorro - que compreende os distritos de Socorro, Cidade Dutra e Grajaú - é composta por 15 unidades básicas de saúde.

Assim, verifica-se a necessidade de uma rede de unidades básicas de saúde em número suficiente para atender à população e realizar atendimentos básicos (especialmente atendimento ambulatorial), de forma a constituir uma rede de apoio à atuação do Hospital Geral do Grajaú e encaminhar ao hospital somente indivíduos com problemas de maior gravidade. No caso de atendimentos de urgência realizados sem prévio agendamento de forma espontânea ou encaminhados pelo sistema de referência, observam-se flutuações quanto ao percentual de atendimentos realizados.

A população na região tem apresentado contínuo crescimento em torno de $3 \%$ ao ano, no entanto, a oferta de equipamentos públicos de saúde não tem acompanhado o incremento populacional. Os dados da OSS demonstram que há uma elevada média de atendimentos ambulatoriais, urgência e SADT por mil habitantes no hospital.

Dada a localização do Hospital Geral do Grajaú em área com significativo déficit de Unidades Básicas de Saúde, verifica-se que o perfil de atendimento foi modificado para se adaptar às condições de infraestrutura de saúde da região, assim, alguns serviços ofertados, como atendimento de urgência/emergência e atendimento ambulatorial, apresentam-se bastante sobrecarregados pela alta demanda.

0 alto número de internações na área pediátrica e ginecológica/obstétrica apresentam-se em acordo com o perfil sócio-demográfico populacional da região. A adequação dos serviços de saúde à demanda local tem sido realizada através da flexibilidade administrativa que a OSS possui para contratação de recursos humanos e aquisição de insumos.

\section{Considerações Finais}

No que tange à gestão do Hospital Geral do Grajaú, é inquestionável a maior flexibilidade de que dispõem as Organizações Sociais, em termos de recursos humanos e recursos materiais, de forma a buscar adaptação ao ambiente em que está inserida. Tal fato ocorre devido às características do regulamento de compras e contratos utilizados pelas Organizações Sociais, que não são sujeitos à Lei Geral de Licitações e ao Sistema de Administração de Serviços Gerais da União, como na administração estatal, sendo uma ferramenta a ser elaborada em cada organização.

A flexibilidade administrativa da OSS engloba, além da autonomia para definição de procedimentos de compras, a autonomia de criação de uma estrutura interna e uso de orçamento, desde que respeitados os limites de empenho dos recursos na administração de pessoal.

A política adotada para gestão de recursos humanos é um importante instrumento das OSS, sendo apontados por diversos estudos como um dos fatores de maior destaque no novo padrão de administração de hospitais públicos, uma vez que as OSS inserem práticas de incentivos típicas do setor privado na administração dos hospitais públicos. Tais características possibilitam maior eficiência na gestão por resultados, comparativamente à administração estatal. A flexibilidade em relação às compras também constitui uma ferramenta essencial na gestão via OSS, pois amplia a capacidade de resposta da organização às mudanças nas demandas em saúde. 
Entretanto, a afirmação de que o modelo de Organizações Sociais de Saúde possui maior flexibilidade administrativa pode ser questionada, tendo em vista que as limitações ao empenho do orçamento com recursos humanos associadas ao estabelecimento de metas e resultados no contrato de gestão permitem pouca flexibilidade administrativa, em última instância.

O modelo de parceria entre Organizações Sociais e Estado enfrenta diversas críticas quanto à possibilidade de constituir somente um mecanismo de “privatização dissimulada” (Pinto, 2000 p. 47), já que, através das parcerias, o Estado transfere a responsabilidade da prestação de serviços essenciais às OSS, o que caracterizaria diminuição da atuação efetiva do Estado. Contudo, analisando o modelo adotado através da ótica do agente-principal, verifica-se a necessidade de forte presença do Estado na regulação e fiscalização, assim como desenho institucional adequado à operacionalização do modelo. Ou seja, o Estado teria possibilidade real de obter resultados melhores comparativamente à estrutura de mercado, caso haja uma clara definição dos papéis de cada estrutura que compõe o modelo, mantendo-se um mecanismo de accountability efetivo (Przeworski, 1999).

Portanto, o argumento fortalece a concepção de que não é suficiente a criação de ferramentas de controle das atividades das Organizações Sociais pelo Estado, se o próprio Estado não estiver bem estruturado com funcionários comprometidos com interesses públicos e aptos a fortalecer a capacidade gerencial do Estado, assim como buscar garantir a participação efetiva da sociedade civil.

No entanto, deve-se ressaltar que a introdução do instrumento de contrato de gestão na lógica da administração pública constitui a maior contribuição do modelo gerenciado por Organizações Sociais de Saúde, tendo em vista a consolidação da gestão por resultados como abordagem tipicamente marcada pela busca por eficácia e eficiência na construção de políticas públicas. O contrato de gestão pode ser considerado, ainda, instrumento que permitiu uma importante mudança de paradigma da atuação estatal junto à sociedade, pois possibilitou o fortalecimento do papel regulador e gerenciador de políticas públicas exercido pelo Estado.
Ademais, ressalta-se que a OSS mantenedora do Hospital Geral do Grajaú atende satisfatoriamente à demanda da população de sua região de abrangência, utilizando-se da flexibilidade administrativa na alocação de recursos e, principalmente, na formação do quadro de profissionais de saúde.

\section{Referências}

BARBAN, V. (Org.). A luta pela melhoria da assistência à saúde no Grajaú: avanços e desafios para pautar o orçamento público. São Paulo: Instituto Pólis, 2007. Disponível em: <www. polis.org.br/utilitarios/editor2.o/UserFiles/File/ pesquisa\%2osaúde\%2ograjau.pdf $>$. Acesso em: 20 mar. 2008.

BAZILLI, R. R.; MONTENEGRO, L. S. B. Apontamentos sobre a reforma administrativa. São Paulo: Unesp, 2003.

BRASIL. Constituição da República Federativa do Brasil. Brasília, DF: Senado Federal, 1988.

BRESSER-PEREIRA, L. C. Do Estado patrimonial ao gerencial. In: SACHS, I.; WILHEIM, J.; PINHEIRO, P. S. (Ed.). Brasil: um século de transformações. São Paulo: Companhia das Letras, 2001. p. 223-259.

BRESSER-PEREIRA, L. C. O modelo estrutural de gerência pública. Revista de Administração Pública, Rio de Janeiro, v. 42, n. 2, p. 391-410, 2008.

CARNEIRO JR, N.; ELIAS, P. E. A reforma do Estado no Brasil: as organizações sociais de saúde.

Revista de Administração Pública, Rio de Janeiro, v. 37, n. 2, p. 201-226, 2003.

COSTA, N. do R.; MELO, M. A. B. C. Reforma do Estado e as mudanças organizacionais no setor saúde. Ciência e Saúde Coletiva, Rio de Janeiro, v. 3, n. 1, p. 52-67, 1998.

DATASUS. Cadastro nacional de estabelecimentos de saúde: quadro dos funcionários por especialidade/função no Hospital Geral do Grajaú. Brasília, 2008. Disponível em: <http://cnes.datasus.gov.br/ Mod_Profissional.asp?Navegacao=Proxima\&VCo Unidade=3550302077671>. Acesso em: 11 nov. 2008. 
FUNDAÇÃO GETÚLIO VARGAS; ORGANIZAÇÃO

DAS NAÇÕES UNIDAS PARA A EDUCAÇÃO,

A CIÊNCIA E A CULTURA. Estudos de

contratualização de hospitais no âmbito do SUS.

São Paulo, 2008. Relatório técnico.

FUNDAÇÃO SEADE. Demografia: população, por sexo, segundo grupos de idade no município de São Paulo 2004 a 2007. São Paulo, 2008.

Disponível em: <http://www.seade.gov.br/ produtos/anuario/2003/dem/dem2003_02.xls>.

Acesso em: 20 ago. 2008.

GOMES, M. C. Organizações sociais: a experiência da Secretaria de Estado da Saúde de São Paulo. In: LEVY, E.; GRAGO, P. A. (Org.). Gestão pública no Brasil contemporâneo. São Paulo: Fundap, 2005. p. 164-184.

IBANEZ, N. et al. Organizações sociais de saúde: o modelo do Estado de São Paulo. Ciência e Saúde Coletiva, Rio de Janeiro, v. 6, n. 2, p. 391-404, 2001.

MODESTO, P. E. G. Reforma administrativa e marco legal das organizações sociais no Brasil: as dúvidas dos juristas sobre o modelo das organizações sociais. Revista do Serviço Público, Brasília, DF, v. 48, n. 2, p. 27-57, 1997.

PACHECO, R. S. Contratualização de resultados no setor público: a experiência brasileira e o debate internacional. In: CONGRESO INTERNACIONAL DEL CLAD SOBRE LA REFORMA DEL ESTADO Y DE LA ADMINISTRACIÓN PÚBLICA, 9., 2004, Madrid. Anais... Caracas: CLAD, 2004.
PINTO, E. G. Organizações sociais e reforma do Estado no Brasil: riscos e desafios nesta forma de institucionalizar a parceria Estado-Sociedade organizada. In: CONCURSO DE ENSAYOS DEL CLAD "ADMINISTRACIÓN PÚBLICA Y CIUDADANÍA”, 14., 200o, Caracas. Anais... Caracas: CLAD, 2000.

PRZEWORSKI, A. O Estado e o cidadão. In: BRESSER-PEREIRA, L. C.; WILHEIM, J.; SOLA, L. (Org.). Sociedade e Estado em transformação. São Paulo: Unesp; Brasília, DF: ENAP, 1999. p. 323-359.

RINNE, J. Mejorando el desempeño del sector salud en Brasil: comparación entre distintos modelos de hospitales. Revista del CLAD Reforma y Democracia, Caracas: CLAD, v. 37, p. 101-140, 2007. Disponível em: <htpp://www.clad.org.ve/ fulltext/o052630.pdf>. Acesso em: 8 maio 2008.

SARTI, F. M.; CAMPINO, A. C. C. Fundamentos de economia da saúde e farmacoeconomia. In: NITA, M. E. et al (Org.). Avaliação de tecnologias em saúde: evidência clínica, análise econômica e análise de decisão. Porto Alegre: Artmed, 2010. p. 225-236.

SÃO PAULO (Estado). Lei Complementar no 846, de 4 de junho de 1998. Dispõe sobre a qualificação de entidades como organizações sociais e dá outras providências. Lex: Coletânea de Legislação e Jurisprudência, São Paulo, v. 62, p. 906-913, 1998.

SÃO PAULO. Secretaria Estadual da Saúde. Relatório com os recursos financeiros e produção contratada e realizada pelo hospital no período de 2004 a 2007. São Paulo, 2008. 